\title{
Endoscopic intraperitoneal subserosal dissection combined with endoscopic ultra- sonography-assisted location of an extraluminally growing stromal tumor
}

A 70-year-old gentleman was admitted with a suspected gastric submucosal tumor (SMT) detected by abdominal contrast computed tomography (CT) ( $\triangleright$ Fig.1) performed to investigate his complaints of dull pain in the abdomen. Preoperative endoscopic ultrasonography (EUS) helped to confirm and locate the lesion after repeated esophagogastroduodenoscopy revealed nothing ( Fig.1). Endoscopic intraperitoneal subserosal dissection technique was employed in this case. Details of the steps are shown in > Fig. 2 and $>$ Video $\mathbf{1}$, including mucosal incision, submucosal tunneling, intraperitoneal subserosal dissection, removal of the lesion, and mucosal closure. The pathological diagnosis was gastrointestinal stromal tumor (GIST) (WHO, Digestive System Tumours, 5 th edn.), prognostic group 1. Intravenous antibiotics were given for 1 day after operation. The gastric tube was removed on postoperative day (POD) 2, fluid diet was resumed on POD3, and on POD7 the patient was discharged.

For smaller SMTs deriving from muscularis propria, endoscopic full-thickness resection (EFTR) has comparable feasibility, safety, and effectiveness to surgical resection and, what's more, with less invasiveness, quicker recovery, and lower costs [1, 2]. However, for SMTs with a predominantly extraluminal growth pattern, EFTR is sometimes difficult to perform because of the high mobility of the tumor and the limited view from the gastric cavity. The submucosal tunneling endoscopic resection technique is suitable for predominantly extraluminally growing SMTs [3], but its use is sometimes limited by the location of the lesion. When the tumor lies in an angled position such as the lesser curvature of the antrum, as in the present case, or the greater curvature of the corpus, difficulty arises in creating a submucosal tunnel towards it. To overcome these disadvantages, our recently reported new technique, called endo-
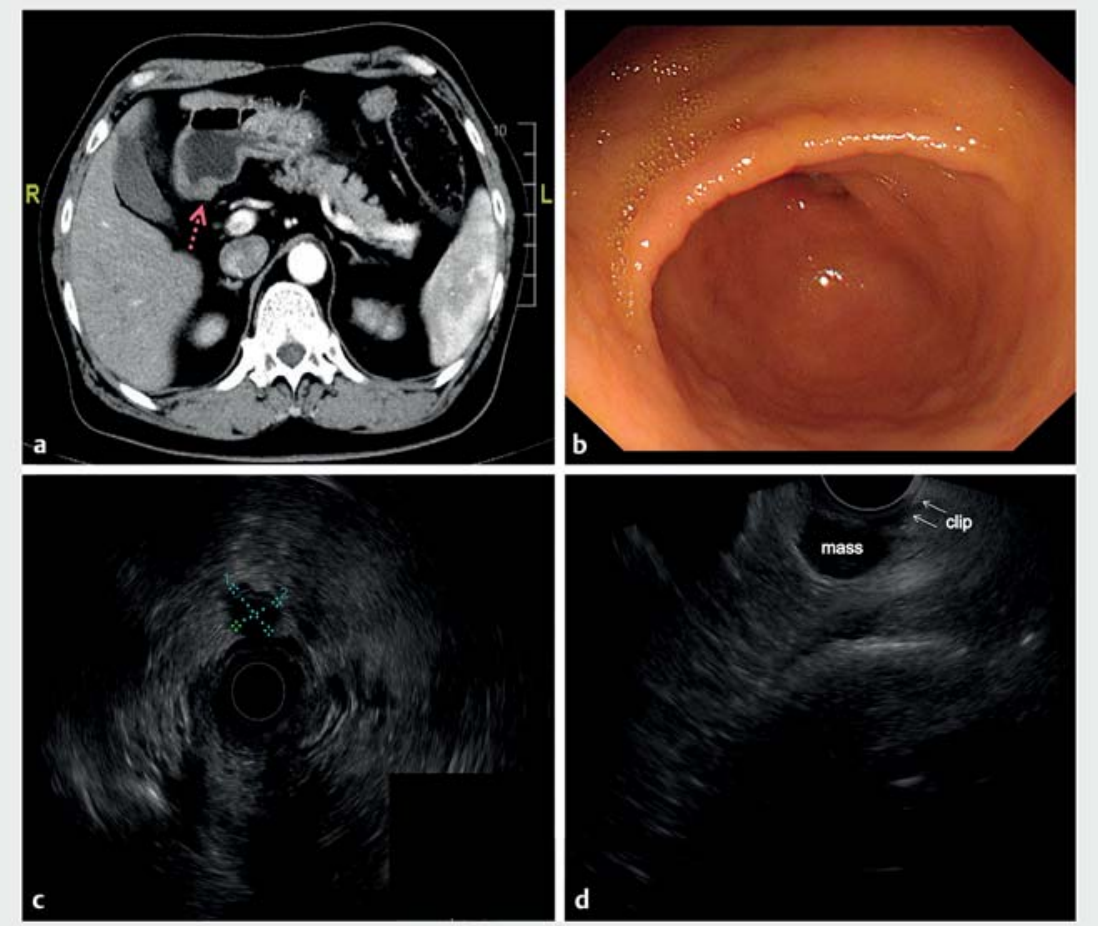

- Fig. 1 a-d Preoperative assessment of the lesion. a Detection of a suspected gastric submucosal tumor on the posterior wall by abdominal contrast computed tomography. b No lesion was found on repeated esophagogastroduodenoscopy after the stomach was sufficiently inflated. c Detection of a hypoechoic mass deriving from the muscularis propria layer, with a predominantly extraluminal growth pattern as shown by radial endoscopic ultrasonography (EUS), $12 \times 10 \mathrm{~mm}$ in size. $\mathbf{d}$ A clip was anchored under linear EUS guidance.

scopic intraperitoneal subserosal dissection, offers a potential solution in such a case [4]. How to locate the lesion precisely also remained a problem, when the small tumor could not be observed under esophagogastroduodenoscopy. CT provides little reference, for the anatomic landmarks in tomographic imaging differ from those under esophagogastroduodenoscopy, and the relative position would alter depending on the gastric inflation status. Subsequently, EUS-guided clip anchoring [5] was attempted and proved highly successful. In conclusion, endoscopic intraperitoneal subserosal dissection on the basis of EUS-guided anchoring is an effective solution for treat- ing SMTs with a predominantly extraluminal growth pattern which are invisible under esophagogastroduodenoscopy.

Endoscopy_UCTN_Code_CCL_1AB_2AG_3AB

Funding

Science and Technology Commission of Shanghai Municipality

http://dx.doi.org/10.13039/

501100003399

19441905200

National Natural Science Foundation of China

http://dx.doi.org/10.13039/

501100001809

82003074 

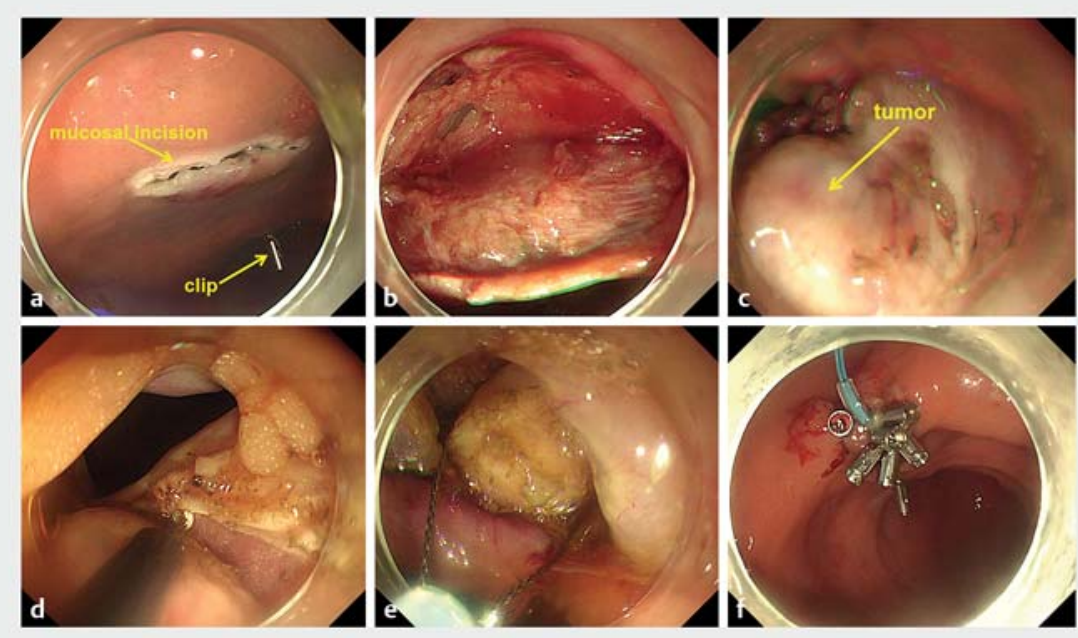

- Fig. 2 a-f Endoscopic intraperitoneal subserosal dissection for a 70-year-old gentleman. a Mucosal incision on the oral side of the marked site. $\mathbf{b}$ Creation of a short submucosal tunnel and incision of the muscularis propria in order to enter the abdominal cavity. $\mathbf{c}$ Exposure of the tumor from covering omentum. $\mathbf{d}$ Dissection of the tumor from the serosa and underlying muscularis propria. e Retrieval of the tumor into the gastric cavity by a snare. $\mathbf{f}$ Closure of the incision using a purse suture.

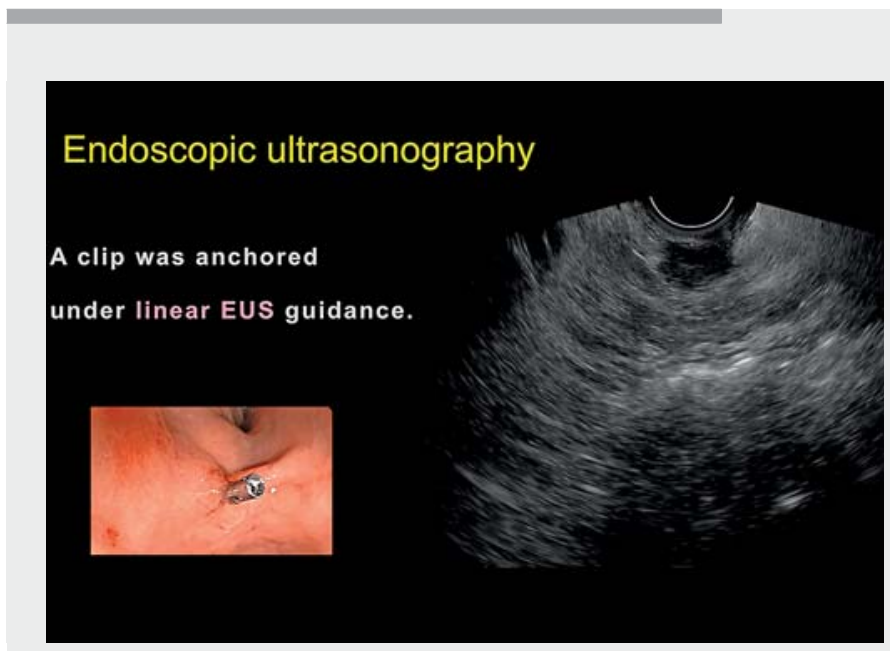

$\checkmark$ Video 1 Endoscopic intraperitoneal subserosal dissection after endoscopic ultrasonography-assisted location of an extraluminally growing stromal tumor in a 70-year-old gentleman.

Shanghai "Rising Stars of Medical Talent" Youth Development Program (Youth Medical Talents - Specialist Program) SHWJRS (2021)-99

Shanghai Municipal Education Commission http://dx.doi.org/10.13039/

501100003395

$18 C G 07$

\section{Competing interests}

The authors declare that they have no conflict of interest.
The authors

Meng-Jiang $\mathrm{He}^{1}{ }^{*}$, Wei-Feng Chen ${ }^{2}$, Xin-Yang Liu $^{1}$, Jie $\mathrm{He}^{2}$, Jian-Wei Hu${ }^{1}$, Ping-Hong Zhou ${ }^{1}$

1 Endoscopy Center and Endoscopy Research Institute, Zhongshan Hospital, Fudan University, Shanghai, China

2 Endoscopy Center, Zhongshan Hospital, Fudan University (Xiamen Branch), Xiamen, Fujian, China

\section{Corresponding author}

\section{Ping-Hong Zhou, MD}

Endoscopy Center and Endoscopy Research Institute, Zhongshan Hospital, Fudan University, Shanghai, China ph.zhou@yahoo.com

\section{References}

[1] Koh YX, Chok AY, Zheng HL et al. A systematic review and meta-analysis comparing laparoscopic versus open gastric resections for gastrointestinal stromal tumors of the stomach. Ann Surg Oncol 2013; 20: 3549-3560

[2] Liu S, Zhou X, Yao Y et al. Resection of the gastric submucosal tumor (G-SMT) originating from the muscularis propria layer: comparison of efficacy, patients' tolerability, and clinical outcomes between endoscopic fullthickness resection and surgical resection. Surg Endosc 2020; 34: 4053-4064

[3] Cai MY, Zhu BQ, Xu MD et al. Submucosal tunnel endoscopic resection for extraluminal tumors: a novel endoscopic method for en bloc resection of predominant extraluminal growing subepithelial tumors or extragastrointestinal tumors (with videos). Gastrointest Endosc 2018; 88: 160-167

[4] Liu X, Chen T, Cheng J et al. Endoscopic transmural route for dissection of gastric submucosal tumors with extraluminal growth: experience in two cases. Gut 2021; 70: 2052-2054

[5] Cao L, Chen N, Chen Y et al. Foreign body embedded in the lower esophageal wall located by endoscopic ultrasonography: a case report. Medicine (Baltimore) 2018; 97: 26 (e11275)

\section{Bibliography}

Endoscopy 2022; 54: E538-E539

DOI 10.1055/a-1675-1699

ISSN 0013-726X

published online 15.11 .2021

(c) 2021. Thieme. All rights reserved.

Georg Thieme Verlag KG, Rüdigerstraße 14,

70469 Stuttgart, Germany

* These authors share first authorship. 